\title{
One-pot Hydrothermal Synthesis of ZnO Microspheres/Graphene Hybrid and its Electrochemical Performance
}

\author{
Ye Lin ${ }^{l}$, Hongdong Liu ${ }^{1, *}$, Zhongli Hu ${ }^{1}$, Rong Hu ${ }^{1}$,Haibo Ruan ${ }^{1}$, Lei Zhang ${ }^{2, *}$ \\ ${ }^{1}$ Research institute for new materials technology, Chongqing university of arts and sciences, \\ Chongqing 402160, PR China \\ ${ }^{2}$ College of life science, Chongqing normal university, Chongqing 401331, PR China \\ "E-mail: $\underline{\text { hd0415@126.com, leizhang0215@126.com }}$
}

doi: $10.20964 / 2016.09 .04$

Received: 29 May 2016 / Accepted: 11 July 2016 / Published: 7 August 2016

In this paper, the $\mathrm{ZnO}$ microspheres/graphene hybrids were successfully prepared from zinc acetate and GO aqueous solution by a facile one-pot hydrothermal method without any surfactant. The assynthesized samples were characterized by X-ray diffraction (XRD), field emission scanning electron microscope (FESEM), thermogravimetric (TGA) analysis, nitrogen adsorption/desorption isotherms and pore size distribution. When evaluated as anode material for lithium ion batteries, it delivered a high initial discharge capacity of $1150 \mathrm{mAh} \mathrm{g}^{-1}$ and exhibited excellent rate performance at different current densities.

Keywords: $\mathrm{ZnO}$ microspheres/graphene hybrid, anode materials, lithium ion batteries, one-pot hydrothermal.

\section{FULL TEXT}

(C) 2016 The Authors. Published by ESG (www.electrochemsci.org). This article is an open access article distributed under the terms and conditions of the Creative Commons Attribution license (http://creativecommons.org/licenses/by/4.0/). 